\title{
The Safety and Feasibility of Bilateral Radial Approach in Chronic Total Occlusion Percutaneous Coronary Intervention
}

\author{
Chiung-Jen Wu, ${ }^{1}$ MD, Hsiu-Yu FAng,${ }^{1 *}$ MD, Cheng-I Cheng, ${ }^{1}$ MD, Hesham Hussein,,${ }^{1,2}$ MD, \\ Sayed M Abdou, ${ }^{1,2}$ MD, Ali A Youssef, ${ }^{3}$ MD, Anuj Bhasin, ${ }^{1}$ MD, Cheng-Hsu Yang, ${ }^{1}$ MD, \\ Chien-Jen Chen, ${ }^{1}$ MD, Yuan-Kai HsieH, ${ }^{1} \mathrm{MD}$, Hon-Kan YIP,${ }^{1 \S} \mathrm{MD}$, and Chih-Yuan FAnG, ${ }^{1} \mathrm{MD}$
}

\begin{abstract}
SUMMARY
Few studies have reported results for transradial (TR) percutaneous coronary intervention (PCI) for chronic total occlusion (CTO) lesions. The purpose of this study was to evaluate the feasibility and safety of bilateral radial PCI for CTO lesions.

Eighty-five consecutive patients with CTO lesions received PCI via a bilateral TR approach. A high radial artery puncture (10-15 cm above styloid process) accommodating a 7 Fr catheter ( $85 \mathrm{~cm}$ long) was used for a retrograde approach, and a 6 Fr catheter was used in the other radial artery for an antegrade approach. Retrograde wiring was conducted primarily or after failure of antegrade wiring. Mean duration of CTO was $42.8 \pm 54.9$ months. Vessels with occlusions attempted were the left anterior descending artery $(40.0 \% ; 34 / 85)$, right coronary artery $(58.8 \% ; 50 / 85)$, and left circumflex artery (1/85). PCI re-attempts were made in $41.2 \%$ of the cases. The overall success rate was $87.1 \%$. Retrograde wiring was successful in 61/85 cases $(71.8 \%)$, via septal collaterals followed by epicardial collaterals and saphenous vein graft. There were no major complications (30 day in-hospital death, $Q$ wave myocardial infarction, or emergency bypass surgery), or serious access site complications.

For experienced TR-PCI operators who are already doing complex TR coronary interventions, the bilateral radial approach for CTO lesions appears feasible and safe. (Int Heart J 2011; 52: 131-138)
\end{abstract}

Key words: Antegrade, Chronic total occlusion, Percutaneous coronary intervention, Retrograde

$\mathrm{T}$ he most challenging lessons learned in the recent era for interventional cardiologists may have come from the use of percutaneous coronary intervention (PCI) for chronic total occlusion (CTO). Successful recanalization of CTO in patients with viable myocardium may reduce angina symptoms, decrease the need for bypass surgery, and may improve long-term survival. ${ }^{1-3)} \mathrm{PCI}$ instruments and the techniques for CTO lesions have improved over time, ${ }^{4)}$ and excellent outcomes have been achieved in multiple pilot studies, especially with the combined use of drug-eluting stents. ${ }^{5}$ )

The most significant improvement in PCI for CTO of coronary vessels has been the introduction of the retrograde approach via bypass grafting ${ }^{8)}$ and epicardial collaterals. Septal collaterals have also been utilized in the treatment of CTO lesions. ${ }^{9)}$ The combined use of the traditional antegrade approach and the newly-developed retrograde approach has markedly increased the overall success rate, while not increasing the rate of major complications. ${ }^{10-14)}$

The transfemoral arterial approach (TFA) is the worldwide accepted method for CTO interventions because the artery can accommodate a larger guiding catheter, and hence provides better support and a larger working space. However, the conventional TFA has several clinical and anatomical limitations including distal abdominal aortic disease, very tortuous or occluded ilio-femoral arteries, morbid obesity, and long period of bed rest required after the procedure. Significant hemostatic difficulties can occur with the TFA in obese patients, which may increase the risk of groin complications, ${ }^{15)}$ and lead to longer hospitalization. An alternative approach for CTO interventions that addresses these limitations is therefore needed.

The transradial approach (TRA) for PCI was introduced in 1993, and growing evidence has shown its benefits to include earlier ambulation, shorter hospital stay, lower medical cost, fewer access site complications, and patient preference. The popularity of the TRA for PCI varies with country, and although a longer learning curve of TRA has been reported, in places such as Canada, Europe, and Asian regions the TRA for PCI comprise from $40 \%$ to $60 \%$ of all PCI cases. The usefulness of the TRA for complex PCIs such as for unprotected left main disease, or stenting for carotid artery stenosis, has been proven by other studies of its feasibility and safety. ${ }^{16,17)}$ An acceptable success rate of the TRA for CTO lesions has also been published in several case reports.

The aim of the present pilot study was to evaluate the

From the ${ }^{1}$ Division of Cardiology, Department of Internal Medicine, Chang Gung Memorial Hospital- Kaohsiung Medical Center, Chang Gung University College of Medicine, Kaohsiung, Taiwan, R.O.C., ${ }^{2}$ National Heart Institute, Cairo, and ${ }^{3}$ Department of Cardiology, Suez Canal University Hospital, Ismailia, Egypt.

Indicates equal contribution as first author.

${ }^{\S}$ Indicates equal contribution as correspondence author.

Address for correspondence: Chih-Yuan Fang, MD, Division of Cardiology, Department of Internal Medicine, Chang Gung Memorial Hospital-Kaohsiung Medical

Center, Chang Gung University College of Medicine, 123, Ta Pei Road, Niao Sung Hsiang Kaohsiung Hsien 83301, Taiwan, R.O.C.

Received for publication December 6, 2010.

Revised and accepted February 21, 2011. 
safety and feasibility of PCI via a bilateral forearm approach (transradial, transbrachial, and transulnar) in patients with coronary CTO lesions.

\section{MeThodS}

The transradial (TR) PCI program was begun at our institution in 1994, and the TRA became our preferred method (> $95 \%$ ) for routine PCIs since the year 2000. Each subject provided written informed consent, and the study protocol was approved by the hospital's Internal Research Board committee. Patient enrollment and exclusion criteria: A diagram of the study enrollment is shown in Figure 1. Enrollment criteria were a documented CTO lesion which was defined as thrombolysis in myocardial infarction grade (TIMI) of 0 for more than 3 months, with the presence of typical angina or reversible myocardial ischemia in a thallium stress study. Exclusion criteria were: 1) a history of acute or recent stroke ( $<2$ months), 2) acute or recent myocardial infarction (MI), 3) surgery or trauma within the preceding 2 months, 4) unfavorable anatomy for the radial approach such as subclavian or innominate artery tortuosity and/or stenosis, and 5) positive Allen's test in both arms. From May 2006 to May 2010, a total of 85 consecutive patients with CTO of coronary vessels were enrolled prospectively and underwent PCI via an antegrade/retrograde approach.

Definitions: CTO was defined as described earlier. The duration of occlusion was estimated by the history of angina, history of MI in the same territory, or previous angiography. Procedure success was defined as successful guidewire and balloon crossing, with or without final stenting, the presence of a final TIMI 3 flow, and a residual diameter stenosis of $<30 \%$. Contrast-induced acute kidney injury (CIAKI) was defined as an increase of baseline serum creatinine concentration of at least $0.5 \mathrm{mg} / \mathrm{dL}$, or at least $25 \%$, within 48 to 72 hours after exposure to contrast media. Major adverse cardiac events (MACE) were defined as death, MI (Q or non-Q wave), or urgent revascularization during the same admission. Urgent revascularization was defined as emergency bypass surgery, or repeat PCI of the same target vessel within 24 hours. Retrograde success was defined as successful passage of the guidewire through collaterals to the distal part of the CTO vessel, without damage to the collaterals such as spasm or perforation.

Procedure and protocol, selection of puncture site: Both radial arteries were evaluated by either Allen's test or Doppler ultrasonography before the procedure. The larger vessel was selected for the high radial arterial (T-HRA) approach, using a $7 \mathrm{Fr}$ guiding catheter. The other radial was selected for the ordinary TRA using a 6 Fr guiding catheter. The T-HRA approach was defined as a puncture site $10 \mathrm{~cm}$ above the styloid process. ${ }^{17)} \mathrm{A}$ 7 Fr and a 6 Fr arterial sheath were then inserted into the radial arteries following successful puncture. A transbrachial arterial approach (TBA) or transulnar arterial access (TUA) was utilized for patients either taller than $175 \mathrm{~cm}$, or with one or more of the following: diameter of both radial arteries too small, weak or absent pulsations at the high-radial level, or an ulnar artery diameter larger than the radial artery diameter. A solution consisting of a mixture of $200 \mu \mathrm{g}$ nitroglycerin and 5,000 $\mathrm{U}$ heparin was given intra-arterially via the sheath.

Selection of guiding catheter: A 6 Fr Ikari IL 3.5 guiding cath- eter (Terumo, Tokyo) or a 6 Fr Kimny Mini-radial guiding catheter (Boston Scientific, Grove, MN, USA) were the catheters primarily used in the antegrade approach. A 7 Fr $85 \mathrm{~cm}$

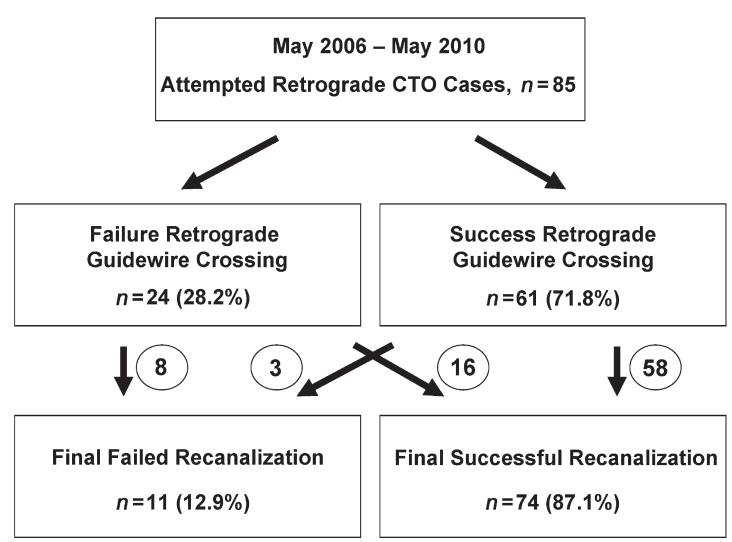

Figure 1. Flowchart of patient enrollment.

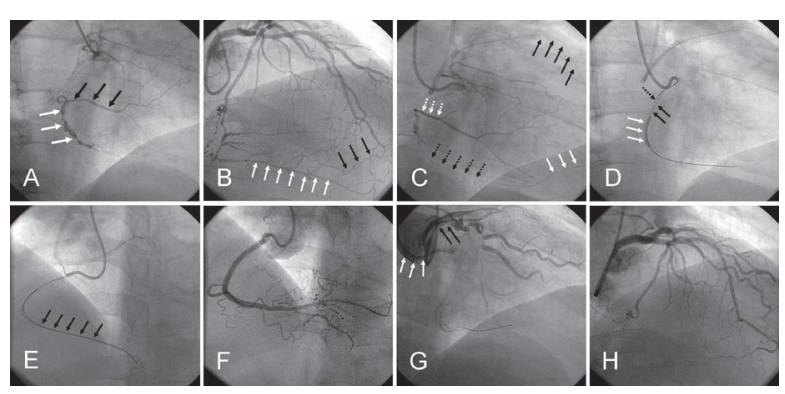

Figure 2. An example of successful controlled antegrade and retrograde subintimal tracking (CART) technique, complicated by donor vessel (left main) dissection. A) Baseline angiogram of the right coronary artery (RCA) revealed chronic total occlusion (CTO) at the proximal RCA (6 Fr AL1 guiding catheter). The mid- to distal RCA (white arrows) was supplied by intracoronary collaterals (black arrows). B) Baseline angiogram of the left anterior descending (LAD) artery and left circumflex artery (LCX) showed patent vessels with multiple septal collaterals (black arrows) to the distal RCA and posterior descending artery (PDA) (white arrows). C) With the support of a transit microcatheter (Cordis, USA), a Fielder FC guidewire (Asahi, Japan) was successfully passed from the LAD (black arrows) through selected septal branches (white arrows) to the PDA (black dotted arrows). The guidewire tip in the true lumen was confirmed by antegrade contrast injection (white dotted arrows). D) Both the retrograde wire (Miracle 6g; Asahi, Japan) (black arrows) and antegrade wire (Miracle 6g) (black dotted arrows) were advanced into the false lumen at the proximal RCA. CART was performed by subsequent retrograde balloon dilatation (Maverick $2.0 \times 20 \mathrm{~mm}$; Boston Scientific, USA) at $14 \mathrm{~atm}$ (white arrows). E) The antegrade wire (Conquest 9g; Asahi, Japan) was successfully advanced to the RCA true lumen (black arrows). F) Final result after 3 drug-eluting stents (Taxus Liberte $2.75 \times 25 \mathrm{~mm}, 3.0 \times 32 \mathrm{~mm}$, and $3.0 \times 32 \mathrm{~mm}$; Boston Scientific, USA) were deployed from the distal RCA to the proximal RCA under intravascular ultrasound (IVUS) guidance. G) Unfortunately, the final check of the left coronary artery created a left main (LM) dissection (black arrows) with the 7 Fr BL3.5 guiding catheter (Terumo, Japan), which extended up to the ascending aorta (white arrows). H) After Tstenting the LM-LCX (Zeta $4.0 \times 38 \mathrm{~mm}$; Abbott, USA) and LAD (Taxus Liberte $3.0 \times 20 \mathrm{~mm}$ ) and final kissing technique, the LAD and LCX showed normal flow with a good angiographic result. (A, C-D, G, right anterior oblique view; B, H, right anterior oblique cranial view; E-F, left anterior oblique cranial view.) 
long BL 3.5-4 or AL 1-2 short guiding catheter (Terumo) was selected for the retrograde approach according to each individual patient. After successful engagement of both guiding catheters, a second bolus of 5,000 U heparin was given via the catheters.

Bilateral simultaneous coronary injections: At least two perpendicular projections with a biplane cine-machine were obtained, and the most proper view for the CTO intervention was selected by using simultaneous bilateral coronary injections.

Selection of guidewire: Guidewire selection for the antegrade approach was decided by each operator after baseline coronary angiography. The guidewires ranged from those with usual stiffness such as Runthrough Floppy (Terumo, Japan) or Rinato wires (Asahi, Tokyo), hydrophilic wires such as Pilot (Abbott, Abbott Park, IL, USA) or Fielder wires (FC or XT; Asahi), and stepwise harder-tip wires such as Miracle (3, 4.5, 6, 12 gm; Asahi) or Conquest wires (9, 12, or 20/8 gm; Asahi). The selected guidewire for the antegrade approach was placed at the proximal cap of the CTO site with a $1.8 \mathrm{~F}$ microcatheter (Finecross; Terumo). The guidewires used for the retrograde approach were either Fielder-FC or Fielder-XT (Asahi), and were supported with a microcatheter or the smallest profile of

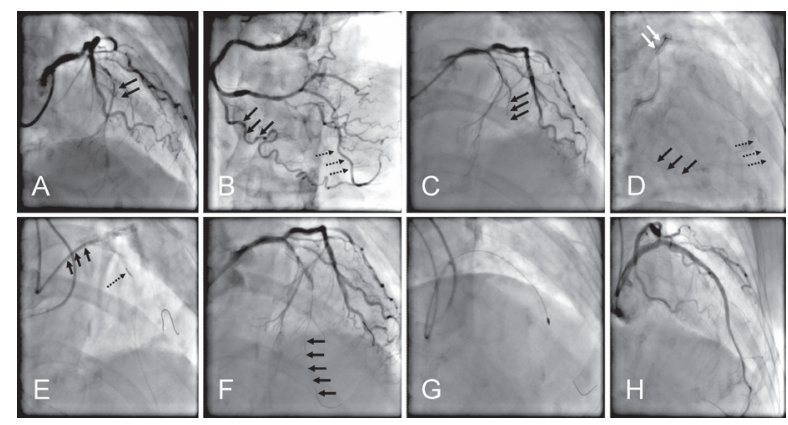

Figure 3. Example of successful retrograde externalization and antegrade rotablation via epicardial collaterals. A) Baseline angiogram of the left anterior descending (LAD) artery showed chronic total occlusion (CTO) at the mid$\mathrm{LAD}$ artery without a visible stump (black arrows), and prior stent at the ostium of the left main (LM) to left circumflex (LCX) artery without in-stent restenosis. B) Baseline angiogram of the right coronary artery (RCA) showed epicardial collaterals from a tortuous right ventricular branch (RV) (black arrows) to the distal LAD artery (black dotted arrows). C) Antegrade approach using a Miracle 3 wire resulted in dissection (black arrows). D) Retrograde approach with a Finecross microcatheter (Terumo, Japan) and Fielder FC guidewire successfully advanced from the right ventricle (RV) branch (black arrows) through an epicardial collateral to distal to the LAD artery true lumen (black dotted arrows). The guidewire was able to pass through CTO segment and then was inserted into the antegrade guiding catheter (white arrows). E) Using a Maverick $2.0 \times 20 \mathrm{~mm}$ balloon anchored to the retrograde wire (black arrows), a Ryujin $1.25 \times 10 \mathrm{~mm}$ over-the-wire balloon (Terumo, Japan) was inflated at the mid- to proximal LAD artery to 16 atm (black dotted arrow). The guidewire was successfully changed to a Rinato $300 \mathrm{~cm}$ guidewire (Asahi, Japan) to allow externalization of the wire out of the antegrade guiding catheter. F) Due to severe calcification of the LAD artery at the occluded segment, a Finecross microcatheter was advanced to the distal LAD artery true lumen, and changed to a 0.009 Rota-wire (black arrows). G) Rotablation was initiated with a $1.5 \mathrm{~mm}$ Rota burr for the calcified CTO lesion. H) After Tstenting with two bare metal stents (Vision $2.5 \times 28 \mathrm{~mm}$ (Abbott, USA), Zeta $3.0 \times 38 \mathrm{~mm}$ ) from the mid-LAD to the ostium of the LAD, and final kissing angioplasty from the LM to both the LAD and LCX arteries, good results were obtained. (A, C-H, right anterior oblique cranial view; $\mathbf{B}$, left anterior oblique cranial view.) over-the-wire balloon (Ryujin $1.25 \times 10 \mathrm{~mm}, 135 \mathrm{~cm}$, or 150 cm; Terumo).

Bilateral forearm approach for CTO intervention, retrograde and antegrade approaches: The reasons for attempting a retrograde approach were determined by the operator, and were typically because of a failure of the antegrade approach (previously or the same procedure), or because of unfavorable anatomy for antegrade wiring. Retrograde routes ranged from septal channel collaterals, epicardial collaterals, saphenous vein grafts (SVG), or the left internal mammary artery (LIMA). Collateral selection was made by further super-selective contrast injection via the microcatheter into the collaterals. Fielder FC or Fielder XT guidewires (Asahi) were carefully passed through the collaterals in a stepwise manner. After the guidewire was successfully advanced into the distal part of the CTO vessel, retrograde wiring was attempted with hydrophilic wires, with subsequent gradual stepping-up of the wire-tip strength, such as with Miracle 3-6 gm or Conquest-pro 9-12 gm wires. Different retrograde methods included the 1) kissing wire technique, 2) controlled antegrade and retrograde subintimal tracking (CART) technique (Figure 2), and 3) reverse CART technique or knuckle wire technique were used alone or in combination. After the retrograde wire had passed the occluded segment, the retrograde wire was manipulated into the antegrade guiding catheter, either for balloon anchoring or for externalization of the wire, by changing the original $190 \mathrm{~cm}$ wire into a $300 \mathrm{~cm}$ long wire through the microcatheter (Figure $3)$.

Stepwise balloon angioplasty was performed via the antegrade or retrograde route, and then an antegrade soft-tip wire was advanced into the distal portion of the occluded vessel along with the retrograde wire, or by using a "Crusade" (Kane$\mathrm{ka}$, Corp) multifunction probing catheter. Next, an intravascular ultrasound assessment of the occluded vessel was performed for preparation of subsequent stenting. If all of the retrograde methods failed, an antegrade approach using a stiffer wire or parallel-wire technique was attempted as the last resort. A Tornus drilling catheter or a Rotablator catheter was considered if the lesion could not be crossed with the balloon un-crossing or balloon popping could not be achieved (Figure 3). After successful balloon dilatation, either a bare-metal stent (BMS) or drug-eluting stent (DES) was deployed, based on the patient's preference. (General health insurance guidelines in Taiwan only reimburse for BMS. The additional cost of a DES has to be paid for by the patient.)

Medications: Patients were pretreated with oral aspirin (100 $\mathrm{mg}$ /day). Patients were also pretreated with a loading dose of clopidogrel (300 or $600 \mathrm{mg}$ ), 4 to 12 hours before the procedure. A postprocedure dose of clopidogrel $(300 \mathrm{mg}$ ) was administered, and then $75 \mathrm{mg} /$ day was prescribed for at least 9 months following implantation of DES, or at least 3 months after BMS deployment

Statistical analysis: Continuous variables are reported as the mean \pm standard deviation, while categorical variables are reported as frequencies. Categorical variables were compared between groups by the chi-square test. A $P$-value of $<0.05$ was considered statistically significant. Statistical analysis was performed using SPSS version 13.0 software (SPSS, Chicago, IL, USA). 


\section{RESUlts}

Patient population: The patient population included 85 patients (mean age, $59.7 \pm 11.1$ years; $94.1 \%$ male) who underwent CTO revascularization using a bilateral forearm access and combined antegrade and retrograde approaches. Table I shows the demographic and baseline clinical data of the 85 patients. The retrograde approach was successful in 61 patients and unsuccessful in 24 patients. The retrograde approach success and failure patients did not differ significantly in body weight, body height, and baseline creatinine level. The incidences of risk factors, such as hypertension, diabetes mellitus, hyperlipidemia, and current smoking were similar in both groups. Forty-one percent of the patients had a history of MI, and $7.1 \%$ of the patients had a history of bypass surgery. The mean left ventricular ejection fraction, as determined by twodimensional echocardiography, was $54.8 \pm 11.3 \%$. The mean duration of CTO was $42.8 \pm 54.9$ months, and the longest duration was 240 months. Table I shows the 3 major reasons for attempting the retrograde approach. Re-attempt after prior antegrade failure accounted for $44.7 \%$ of the cases, CTO morphology unfavorable for the antegrade approach accounted for $45.9 \%$ of the cases, and in $9.4 \%$ of the cases a retrograde approach was attempted immediately after failure of primary antegrade wiring.

Angiographic characteristics and measurements: Table II summarizes data regarding the access site, arterial sheath size, baseline angiographic characteristics, and the procedure outcomes of the 85 patients. The target lesion was in the right cor-

Table I. Baseline Demographic Characteristics

\begin{tabular}{|c|c|}
\hline & All patients $(n=85)$ \\
\hline \multicolumn{2}{|l|}{ Demographics } \\
\hline Age, years & $59.7 \pm 11.1$ \\
\hline Male & $80(94.1)$ \\
\hline Body weight, $\mathrm{kg}$ & $73.0 \pm 11.6$ \\
\hline Body height, $\mathrm{cm}$ & $165.1 \pm 6.0$ \\
\hline Creatinine, $\mathrm{mg} / \mathrm{dL}$ & $1.3 \pm 1.1$ \\
\hline \multicolumn{2}{|l|}{ Risk factors } \\
\hline Diabetes mellitus & $27(31.8)$ \\
\hline Hypertension & $72(84.7)$ \\
\hline Cholesterol > $200 \mathrm{mg} / \mathrm{dL}$ & $19(22.4)$ \\
\hline Current smoking & $41(48.2)$ \\
\hline \multicolumn{2}{|l|}{ Comorbidity } \\
\hline Previous MI & $35(41.2)$ \\
\hline Previous CABG & $6(7.1)$ \\
\hline One-vessel disease & $36(42.4)$ \\
\hline Two-vessel disease & $22(25.9)$ \\
\hline Triple vessel disease & $27(31.8)$ \\
\hline $\operatorname{LVEF}(\%)$ & $54.8 \pm 11.3$ \\
\hline History of CTO, months & $42.8 \pm 54.9$ \\
\hline \multicolumn{2}{|l|}{ Reasons for retrograde approach } \\
\hline Second attempt after prior antegrade failure & $38(44.7)$ \\
\hline CTO morphology unfavorable & $39(45.9)$ \\
\hline Antegrade failure then switch to retrograde & $8(9.4)$ \\
\hline \multicolumn{2}{|l|}{ Retrograde attempt } \\
\hline Retrograde guidewire successful crossing & $61(71.8)$ \\
\hline Retrograde guidewire failed crossing & $24(28.2)$ \\
\hline
\end{tabular}

Data are presented as mean \pm SD or number $(\%)$ of patients. MI indicates myocardial infarction; CABG, coronary artery bypass graft; LVEF, left ventricle ejection fraction; and CTO, chronic total occlusion. onary artery (RCA) in $58.8 \%$ of the cases, and the left anterior descending artery (LAD) in $40 \%$ of the cases. There was only one patient who underwent left circumflex (LCX) CTO intervention. There were 81 patients $(95.3 \%)$ in whom antegrade access via the usual radial access was used, and 6 Fr guiding catheters were used in 77 of these patients. There were 66 patients $(89.4 \%)$ in whom a retrograde approach via high radial access was used, and 7 Fr guiding catheters were used in 68 of the patients $(80 \%)$. There were no differences between patients with retrograde success or failure with respect to access site, arterial sheath size, and the target occluded vessels.

Angiography revealed proximal tortuosity of the occluded vessel in 19 patients $(22.4 \%)$, and a side branch at the occlusion site in 51 patients $(60.0 \%)$. Thirteen patients $(15.3 \%)$ had heavy calcifications, as verified with fluoroscopic examination. However, there was no direct evidence indicating these angiographic characteristics might affect retrograde success. A "blunt stump" at the occlusion site was present in $62.3 \%$ of retrograde success patients, which was higher than that of the retrograde failure patients $(37.5 \%, P=0.038)$ (Table II). As

Table II. Angiographic Characteristics in Retrograde Success and Failure Groups

\begin{tabular}{|c|c|c|c|c|}
\hline & $\begin{array}{c}\text { All } \\
\text { patients } \\
(n=85)\end{array}$ & $\begin{array}{c}\text { Retrograde } \\
\text { Success } \\
(n=61)\end{array}$ & $\begin{array}{l}\text { Retro- } \\
\text { grade } \\
\text { Failure } \\
(n=24)\end{array}$ & $P$ \\
\hline \multicolumn{5}{|l|}{ Target CTO Vessel } \\
\hline LAD & $34(40.0)$ & $22(36.0)$ & $12(50.0)$ & 0.238 \\
\hline RCA & $50(58.8)$ & $39(63.9)$ & $11(45.8)$ & 0.127 \\
\hline LCX & $1(1.2)$ & $0(0)$ & $1(4.2)$ & 0.109 \\
\hline \multicolumn{5}{|l|}{ Antegrade Access Site } \\
\hline Radial & $81(95.3)$ & $57(93.4)$ & $24(100)$ & 0.199 \\
\hline Ulnar & $2(2.4)$ & $2(3.3)$ & $0(0)$ & 0.369 \\
\hline Brachial & $2(2.4)$ & $2(3.3)$ & $0(0)$ & 0.369 \\
\hline \multicolumn{5}{|l|}{ Antegrade Sheath Size } \\
\hline $5 \mathrm{Fr}$ & $4(4.7)$ & $3(4.9)$ & $1(4.2)$ & 0.883 \\
\hline $6 \mathrm{Fr}$ & 77 (90.6) & $55(90.2)$ & $22(91.7)$ & 0.831 \\
\hline $7 \mathrm{Fr}$ & $4(4.7)$ & $3(4.9)$ & $1(4.2)$ & 0.883 \\
\hline \multicolumn{5}{|l|}{ Retrograde Access Site } \\
\hline High Radial & $76(89.4)$ & $53(86.9)$ & $23(95.8)$ & 0.227 \\
\hline High Ulnar & $5(5.9)$ & $4(6.6)$ & $1(4.2)$ & 0.673 \\
\hline Brachial & $4(4.7)$ & $4(6.6)$ & $0(0)$ & 0.199 \\
\hline \multicolumn{5}{|l|}{ Retrograde Sheath Size } \\
\hline $6 \mathrm{Fr}$ & $17(20.0)$ & $11(18.0)$ & $6(25.0)$ & 0.470 \\
\hline $7 \mathrm{Fr}$ & $68(80.0)$ & $50(82.0)$ & $18(75.0)$ & 0.470 \\
\hline \multicolumn{5}{|l|}{ Angiographic Finding } \\
\hline Proximal Tortuosity & $19(22.4)$ & $15(24.6)$ & $4(16.7)$ & 0.430 \\
\hline $\begin{array}{l}\text { Side Branch at } \\
\text { Occlusion }\end{array}$ & $51(60.0)$ & $36(59.0)$ & $15(62.5)$ & 0.768 \\
\hline Heavy Calcification & $13(15.3)$ & $9(14.8)$ & $4(16.7)$ & 0.825 \\
\hline \multicolumn{5}{|l|}{ Morphology of Stump } \\
\hline Tapered Stump & $20(23.5)$ & $12(19.7)$ & $8(33.3)$ & 0.181 \\
\hline Blunt Stump & $47(55.3)$ & $38(62.3)$ & $9(37.5)$ & 0.038 \\
\hline No Stump & $18(21.1)$ & $11(18.0)$ & $7(29.2)$ & 0.258 \\
\hline \multicolumn{5}{|l|}{ Collaterals Grade } \\
\hline Grade 1 & $41(48.2)$ & $29(47.5)$ & $12(50.0)$ & 0.838 \\
\hline Grade 2 & $37(43.5)$ & $25(41.0)$ & $12(50.0)$ & 0.450 \\
\hline Grade 3 & $7(8.2)$ & $7(11.5)$ & $0(0)$ & 0.083 \\
\hline Corkscrew Type & $22(25.9)$ & $9(14.8)$ & $13(54.2)$ & $<0.001$ \\
\hline
\end{tabular}

Data are presented as mean \pm SD or number $(\%)$ of patients. CTO indicates chronic total occlusion; LAD, left anterior descending artery; RCA, right coronary artery; LCX, left circumflex artery; and Fr, French. 
Table III. PCI Results in Retrograde Success and Failure Groups

\begin{tabular}{|c|c|c|c|c|}
\hline & $\begin{array}{l}\text { All patients } \\
\quad(n=85)\end{array}$ & $\begin{array}{c}\text { Retrograde } \\
\text { Success } \\
(n=61)\end{array}$ & $\begin{array}{c}\text { Retrograde } \\
\text { Failure } \\
(n=24)\end{array}$ & $P$ \\
\hline \multicolumn{5}{|l|}{ Collaterals Used } \\
\hline Septal Collaterals & $69(81.2)$ & $48(78.7)$ & $21(87.5)$ & 0.350 \\
\hline Epicardial Collaterals & $9(10.6)$ & $6(9.8)$ & $3(12.5)$ & 0.719 \\
\hline Bypass Grafts & $7(8.2)$ & $7(11.5)$ & $0(0)$ & 0.083 \\
\hline \multicolumn{5}{|l|}{ Retrograde Techniques } \\
\hline CART & $25(29.4)$ & $25(41.0)$ & - & - \\
\hline Reverse CART & $7(8.2)$ & $7(11.5)$ & - & - \\
\hline Marker or Kissing Wire & $37(43.5)$ & $37(60.7)$ & - & - \\
\hline Knuckle Wire & $5(5.9)$ & $5(8.2)$ & - & - \\
\hline Externalization & $8(9.4)$ & $8(13.1)$ & - & - \\
\hline IVUS Guided Puncture & $2(2.4)$ & $2(3.3)$ & - & - \\
\hline Total Guidewire Used & $4.9 \pm 1.5$ & $5.0 \pm 1.5$ & $4.6 \pm 1.3$ & 0.868 \\
\hline Tornus Used & $5(5.9)$ & $4(6.6)$ & $1(4.2)$ & 0.673 \\
\hline Rotablation Used & $1(1.2)$ & $1(1.6)$ & $0(0)$ & 0.528 \\
\hline Occlusion Lesion Length & $56.3 \pm 19.3$ & $60.5 \pm 18.5$ & $45.7 \pm 17.5$ & 0.200 \\
\hline Total Procedure Time & $161.5 \pm 54.4$ & $162.5 \pm 56.6$ & $159.2 \pm 49.3$ & 0.327 \\
\hline Fluoroscopy Time & $70.2 \pm 32.3$ & $70.9 \pm 32.1$ & $68.4 \pm 33.4$ & 0.579 \\
\hline Contrast Volume & $440.3 \pm 186.3$ & $413.6 \pm 159.1$ & $508.1 \pm 232.6$ & 0.039 \\
\hline Procedure Success & $74(87.1)$ & $58(95.1)$ & $16(66.7)$ & $<0.001$ \\
\hline
\end{tabular}

Data are presented as mean \pm SD or number $(\%)$ of patients. PCI indicates percutaneous coronary intervention; CART, controlled antegrade and retrograde subintimal tracking; and IVUS, intravascular ultrasound.

Table IV. Major and Access Site Complications in Retrograde Success and Failure Groups

\begin{tabular}{|c|c|c|c|c|}
\hline & $\begin{array}{c}\text { All } \\
\text { patients } \\
(n=85)\end{array}$ & $\begin{array}{c}\text { Retrograde } \\
\text { Success } \\
(n=61)\end{array}$ & $\begin{array}{l}\text { Retro- } \\
\text { grade } \\
\text { Failure } \\
(n=24)\end{array}$ & $P$ \\
\hline \multicolumn{5}{|l|}{ Complications } \\
\hline Cardiac tamponade & $3(3.5)$ & $1(1.6)$ & $2(8.3)$ & 0.132 \\
\hline GI bleeding & $0(0)$ & $0(0)$ & $0(0)$ & 1.000 \\
\hline Contrast-induced AKI & $2(2.4)$ & $1(1.6)$ & $1(4.2)$ & 0.489 \\
\hline Aortic cusp dissection & $1(1.2)$ & $1(1.6)$ & $0(0)$ & 0.528 \\
\hline Donor vessel dissection & $3(3.5)$ & $3(4.9)$ & $0(0)$ & 0.269 \\
\hline Collaterals perforation & $7(8.2)$ & $3(4.9)$ & $4(16.7)$ & 0.076 \\
\hline Perforation needing coil insert & $2(2.4)$ & $2(3.3)$ & $0(0)$ & 0.369 \\
\hline Perforation not needing coil & $2(2.4)$ & $2(3.3)$ & $0(0)$ & 0.369 \\
\hline \multicolumn{5}{|l|}{ Access Site Complications } \\
\hline Forearm ecchymosis & $3(3.5)$ & $1(1.6)$ & $2(8.3)$ & 0.132 \\
\hline Blood transfusion & $0(0)$ & $0(0)$ & $0(0)$ & 1.000 \\
\hline Access site surgery & $0(0)$ & $0(0)$ & $0(0)$ & 1.000 \\
\hline
\end{tabular}

Data are presented as mean \pm SD or number $(\%)$ of patients. PCI indicates percutaneous coronary intervention; $\mathrm{CABG}$, coronary artery bypass graft; GI bleeding, gastrointestinal bleeding; and Contrast-induced AKI, contrast-induced acute kidney injury.

for cases with a "tapered end" or "no stump" at the occlusion site, there was no significant difference between the groups. There was no difference in either grade 1 or grade 2 collaterals between retrograde success or retrograde failure patients, and in all 7 patients with grade 3 collaterals we were able to achieve retrograde wire success. The only significant difference in collaterals between the groups lies in whether they are the corkscrew type or not (retrograde success $14.8 \%$ versus retrograde failure $54.2 \%$, respectively, $P<0.001$ ).
Table V. Clinical and In-Hospital Outcomes in Retrograde Success and Failure Groups

\begin{tabular}{|c|c|c|c|c|}
\hline & $\begin{array}{l}\text { All patients } \\
\quad(n=85)\end{array}$ & $\begin{array}{c}\text { Retrograde } \\
\text { Success } \\
(n=61)\end{array}$ & $\begin{array}{l}\text { Retro- } \\
\text { grade } \\
\text { Failure } \\
(n=24)\end{array}$ & $P$ \\
\hline \multicolumn{5}{|l|}{ In-hospital adverse outcomes } \\
\hline All-cause death & $0(0)$ & $0(0)$ & $0(0)$ & 1.000 \\
\hline Cardiac death & $0(0)$ & $0(0)$ & $0(0)$ & 1.000 \\
\hline Q-wave MI & $0(0)$ & $0(0)$ & $0(0)$ & 1.000 \\
\hline Non-Q-wave MI & $2(2.4)$ & $2(3.3)$ & $0(0)$ & 0.369 \\
\hline Stroke & $0(0)$ & $0(0)$ & $0(0)$ & 1.000 \\
\hline Emergent PCI & $1(1.2)$ & $1(1.6)$ & $0(0)$ & 0.528 \\
\hline Emergent CABG & $0(0)$ & $0(0)$ & $0(0)$ & 1.000 \\
\hline Stent thrombosis & $1(1.2)$ & $1(1.6)$ & $0(0)$ & 0.528 \\
\hline \multicolumn{5}{|l|}{ Clinical outcomes } \\
\hline Hospital stay, days & $4.6 \pm 5.8$ & $4.1 \pm 3.3$ & $5.8 \pm 9.5$ & 0.015 \\
\hline \multicolumn{5}{|l|}{ Learning curve analysis } \\
\hline Initial 25 patient success & $18 / 25(72.0)$ & - & - & 0.008 \\
\hline Later 60 patient success & $56 / 60(93.3)$ & - & - & \\
\hline
\end{tabular}

Data are presented as mean \pm SD or number (\%) of patients. MI indicates myocardial infarction.

PCI techniques and procedure outcomes: Table III summarizes data regarding the retrograde technique and procedure outcomes during PCI. Septal channel collaterals were used primarily (69 patients, $81.2 \%$ ), followed by epicardial collaterals ( 9 patients, $10.6 \%$ ), and bypass grafts ( 7 patients, $8.2 \%$ ). Success was achieved in 7 bypass grafts used for the retrograde route. The marker or kissing wire technique was used in 37 patients $(43.5 \%)$, and CART or reverse CART was used in 25 patients (29.4\%), which comprised $73 \%$ of the total retrograde procedures. The average number of guidewires used, occlusion 
length, total procedure time, and total fluoroscopy time were not different between retrograde success or failure patients. The average contrast volume used in the 85 patients was 440.3 $\pm 186.3 \mathrm{~mL}$, and the retrograde failure group received a significantly larger volume of contrast than the retrograde success group $(508.1 \pm 232.6 \mathrm{~mL}$ versus $413.6 \pm 159.1 \mathrm{~mL}$, respectively, $P=0.039)$. The overall PCI procedure success rate was $87.1 \%$ (74/85). The retrograde success group had a significantly higher success rate than the retrograde failure group $(95.1 \%$ versus $66.7 \%$, respectively, $P<0.001$ ).

In-hospital clinical events and 30-day follow-up: Table IV summarizes data regarding in-hospital cardiac events and access site complications. Three patients experienced cardiac tamponade after PCI, and were treated by percutaneous pigtail catheter drainage. Two patients experienced contrast-induced nephropathy, and both recovered after conservative treatment. One patient experienced aortic cusp dissection during PCI, which was treated with a coronary cover-stent. There were no differences between retrograde success and failure patients with respect to donor vessel dissection, collateral perforation, coronary perforation, and coil embolization or cover-stent usage. There were minimal access site complications; only 3 patients experienced forearm ecchymosis after the PCI procedure, and none of them required emergent blood transfusion or experienced forearm compartment syndrome.

Table V summarizes data regarding the MACE and 30day clinical outcomes of the 85 patients. There were no in-hospital deaths, Q wave MI, stroke, or 30-day emergent CABG, but one patient required an emergent rescue PCI due to acute ( $<24$ hours) in-stent thrombosis. Two patients experienced non-Q wave MIs. One patient had a catheter-tip induced left main dissection, which was complicated with LAD artery perforation and cardiac tamponade. One patient who had an anomalous RCA (originating from the left cusp) experienced RCA ostial dissection which extended to the ascending aorta. The dissection was treated successfully with bare metal stenting. The final result of this patient was acceptable; a limited intramural hematoma at the ascending aorta remained after stenting, but the hematoma had completely resolved as noted on a 3-month computed tomography follow-up study. The mean hospital stay was $4.6 \pm 5.8$ days, and the retrograde failure group (5.8 \pm 9.5 days) had a relatively longer stay than that of the retrograde success group $(4.1 \pm 3.3$ days, $P=0.015)$. There was a significantly lower success rate $(18 / 25,72.0 \%)$ in our early learning period; however, a significantly higher success rate was achieved in a later period $(56 / 60,93.3 \%, P=0.008)$ (Table V).

\section{Discussion}

In the modern era, PCI for CTO lesions with the presence of viable myocardium is well accepted by most cardiovascular interventionists. Newly-developed devices and techniques for CTO intervention have increased the success rate to $50 \%$ to $80 \%$ or higher. ${ }^{18)}$ Overall procedure success is highly dependent on successful antegrade or retrograde wiring across the occlusion. To the best of our knowledge, this is the first report with a significant number of consecutive patients that has evaluated the feasibility of a bilateral forearm approach for CTO intervention. The study results proved the feasibility and safety of the bilateral forearm approach for PCI of CTO lesions, with a high procedure success rate of $87.1 \%$, and a complication rate similar to that reported in studies using the standard femoral approach. ${ }^{7,19)}$

Comparisons of the bilateral forearm approach for CTO intervention: The first retrograde approach for CTO lesions via bypass grafts was reported in 1990, and had a successful revascularization rate of $75 \%{ }^{8)}$ Thereafter, several case reports have described improvements in the technique and the evolving devices. Following the introduction of the CART technique using septal collaterals, the first report to examine the feasibility of the retrograde approach of PCI for CTO intervention was published in $2006 .^{20)} \mathrm{A}$ subsequent report described a high success rate of passing retrograde collaterals $(82 \%)$, and a high final success rate $(84 \%)$ in 45 patients via the femoral approach. ${ }^{19)}$ Comparatively, the present study reported a similar final success rate of $87.1 \%$, but a lower rate of passing retrograde collaterals $(71.6 \%)$. As compared to the present study, two larger CTO interventional studies involving 157 patients ${ }^{10)}$ and 224 patients $^{13)}$ reported similar final success rates of $84.7 \%$ and $90.6 \%$, respectively. Our results also demonstrated that CTO interventions necessitated a learning curve, and it is very important for each operator to be familiar with the different techniques of the retrograde and antegrade approaches.

Comparison of complications with literature: Common complications in retrograde CTO interventions include a higher incidence of collateral channel perforation and a higher incidence of CIAKI, and retrograde CTO interventions are also associated with longer procedure and fluoroscopy times. ${ }^{7)}$ As compared to a previous study that reported a $3.8 \%$ incidence of septal collateral perforation and a 3.2\% incidence of non-Q wave MI, ${ }^{10)}$ our study reported a higher incidence of septal collateral perforation (7.4\%) and a lower incidence of non-Q wave MI $(2.5 \%)$. Although we did use a larger amount of contrast during our CTO interventions (mean $442 \mathrm{~mL}$ versus 293 $\mathrm{mL}$, respectively), there was no significant difference in postprocedure CIAKI between our patients $(2.5 \%)$ and the $1.2 \%$ reported in a multicenter CTO registry of Japan. ${ }^{7)}$ The fluoroscopy time in the present study was relatively longer than that of the Japan CTO registry study (mean 70 minutes versus 45 minutes, respectively); however, none of our 85 patients developed radiation-induced dermatitis. The two studies, however, cannot be equally compared because most of the patients in the Japan CTO registry $(74 \%, 392 / 528)$ underwent PCI only via an antegrade approach. Major in-hospital events, including emergent CABG or PCI, MI, stroke, cardiac tamponade and cardiac death, were similar between the present report and the other CTO studies. ${ }^{7,9-14,19,20)}$

Prediction and prevention of complications: In our series, 7 patients had collateral perforation during PCI and all underwent a retrograde attempt via septal collaterals. Three (3/7, 42.9\%) were corkscrew type collaterals. The reasons for the collateral perforation might be 1) severe angulated, tortuous and corkscrew like collaterals, 2) invisible collaterals or lack of a clear roadmap during guidewire crossing of the collaterals, or 3) the usage of a hydrophilic guidewire such as a Fielder FC wire. There are some key aspects with respect to preventing collateral perforation. First, the operator should be more careful when selecting collaterals that are severely angulated, tortuous, and corkscrew like. Second, the operator should use a super-selection angiogram (microcatheter in collaterals) as a roadmap. 
Third, the operator should advance the hydrophilic guidewire as gently and carefully as possible.

One aortic cusp dissection and 3 coronary donor vessel dissections were found in our study. The aortic cusp dissection in one patient was due to a pre-existing coronary anomaly (right coronary artery CTO that originated from the left coronary cusp). One of the 3 patients $(1 / 3,33.3 \%)$ who suffered from donor vessel dissection had a pre-existing angiographically unstable plaque in the donor vessel (LM) and 3 had donor vessel dissection during an antegrade approach. Several things should be borne in mind to prevent aortic cusp dissection and donor vessel dissection. First, the operator should manipulate the guiding catheter carefully, especially when there is an anomalous origin. Second, the operator should always keep an eye on the donor vessel when initiating antegrade PCI. However, in our series, no correlation between these complications and TRA during CTO intervention was observed.

Forearm intervention, advantages and disadvantages: Although the TFA for CTO intervention is the first choice for most interventional cardiologists, our previous studies have proven the benefits of the TRA in complex coronary interventions. ${ }^{16,21,22)}$ The presence of excellent clinical results support the use of the TRA, and the TFA may be avoided in special situations such as abdominal aortic atherosclerotic disease, severe aortoiliac disease, and previous ilio-femoral bypass graft placement. In bilateral approach CTO interventional studies, only the Japan CTO registry clearly mentioned the access site. ${ }^{7)}$ In most patients in the Japan CTO registry, a femoral access was used for an antegrade approach (87.6\%), and most often a sheath size of 7 Fr was used $(82.0 \%)$. In our study, both antegrade and retrograde access $(95.1 \%$ and $88.9 \%$, respectively) were via the radial arteries. Other forearm arteries (ulnar or brachial artery) were used as an alternative when the radial artery was too small or the patient was taller than $175 \mathrm{~cm}$. The most frequently used arterial sheath sizes in our study were 6 Fr $(91.4 \%)$ for the antegrade approach and $7 \operatorname{Fr}(79.0 \%)$ for the retrograde approach, and both are smaller than those used in the Japan CTO registry study. Although we did use a smaller guiding catheter for antegrade TRA CTO intervention, our final results were equivalent to femoral approach studies. In addition, the down-sizing of the arterial sheath may have the potential benefit of preventing forearm artery endothelial damage. Only 3 patients in our study had forearm ecchymosis, and none required a blood transfusion or vascular surgery. One important finding of the present study is the demonstration of a mean hospital stay of 4.6 days, which is the shortest among all of the published bilateral CTO interventional studies. ${ }^{7,-14,19,20)}$ Shorter hospital stays can lead to a reduction in total medical costs associated with the procedure.

Limitations of the bilateral forearm approach: There are $3 \mathrm{ma}-$ jor limitations of the bilateral forearm approach. 1) Forearm arteries have potential anatomical variations such as looping or small accessory radial arteries, buckling of the innominate artery can occur, or tortuosity of the subclavian artery may be present, 2) Patients with end-stage renal disease may need further protection of the forearm arteries, and 3) The retrograde shorter guiding catheter $(85-90 \mathrm{~cm})$ is too short to reach the coronary ostia in patients who are taller than $175 \mathrm{~cm}$. In our study, the primary size of the antegrade catheter used was a 6 Fr, which may greatly limit the techniques that can be used. A sheathless approach for CTO intervention using 7 Fr guiding catheters in both arms may be a future direction, however, we have limited experience with this in our laboratory. Despite these limitations, the bilateral forearm approach for CTO intervention is safe and effective, especially for experienced TRA operators who are already performing complex PCIs via the radial approach.

Conclusions: The bilateral forearm approach for PCI of CTO lesions appears to be feasible and safe.

\section{REFERENCES}

1. Suero JA, Marso SP, Jones PG, et al. Procedural outcomes and long-term survival among patients undergoing percutaneous coronary intervention of a chronic total occlusion in native coronary arteries: a 20-year experience. J Am Coll Cardiol 2001; 38: 40914.

2. Olivari Z, Rubartelli P, Piscione F, et al. Immediate results and one-year clinical outcome after percutaneous coronary interventions in chronic total occlusions: data from a multicenter, prospective, observational study (TOAST-GISE). J Am Coll Cardiol 2003; 41: 1672-8.

3. Hoye A, van Domburg RT, Sonnenschein K, Serruys PW. Percutaneous coronary intervention for chronic total occlusions: the Thoraxcenter experience 1992-2002. Eur Heart J 2005; 26: 2630-6.

4. Godino C, Sharp A, Carlino M, Colombo A. Crossing CTOs-the tips, tricks and specialist kit that can mean the difference between success and failure. Catheter Cardiovasc Interv 2009; 74: 1019-46.

5. Kandzari DE, Rao SV, Moses JW, et al. Clinical and angiographic outcomes with sirolimus-eluting stents in total coronary occlusions: the ACROSS/TOSCA-4 (Approaches to Chronic Occlusions With Sirolimus-Eluting Stents/Total Occlusion Study of Coronary Arteries-4) trial. JACC Cardiovasc Interv 2009; 2: 97 106.

6. De Felice F, Fiorilli R, Parma A, et al. 3-year clinical outcome of patients with chronic total occlusion treated with drug-eluting stents. JACC Cardiovasc Interv 2009; 2: 1260-5.

7. Morino Y, Kimura T, Hayashi Y, et al. In-hospital outcomes of contemporary percutaneous coronary intervention in patients with chronic total occlusion insights from the J-CTO Registry (Multicenter CTO registry in Japan). JACC Cardiovasc Interv 2010; 3: 143-51.

8. Kahn JK, Hartzler GO. Retrograde coronary angioplasty of isolated arterial segments through saphenous vein bypass grafts. Cathet Cardiovasc Diagn 1990; 20: 88-93.

9. Surmely JF, Katoh O, Tsuchikane E, Nasu K, Suzuki T. Coronary septal collaterals as an access for the retrograde approach in the percutaneous treatment of coronary chronic total occlusions. Catheter Cardiovasc Interv 2007; 69: 826-32.

10. Rathore S, Katoh O, Matsuo H, et al. Retrograde percutaneous recanalization of chronic total occlusion of the coronary arteries: procedural outcomes and predictors of success in contemporary practice. Circ Cardiovasc Interv 2009; 2: 124-32.

11. Hsu JT, Tamai H, Kyo E, Tsuji T, Watanabe S. Traditional antegrade approach versus combined antegrade and retrograde approach in the percutaneous treatment of coronary chronic total occlusions. Catheter Cardiovasc Interv 2009; 74: 555-63.

12. Tsujita K, Maehara A, Mintz GS, et al. Intravascular ultrasound comparison of the retrograde versus antegrade approach to percutaneous intervention for chronic total coronary occlusions. JACC Cardiovasc Interv 2009; 2: 846-54.

13. Kimura M, Katoh O, Tsuchikane E, et al. The efficacy of a bilateral approach for treating lesions with chronic total occlusions the CART (controlled antegrade and retrograde subintimal tracking) registry. JACC Cardiovasc Interv 2009; 2: 1135-41.

14. Rathore S, Katoh O, Tuschikane E, Oida A, Suzuki T, Takase S. A novel modification of the retrograde approach for the recanalization of chronic total occlusion of the coronary arteries intravascu- 
lar ultrasound-guided reverse controlled antegrade and retrograde tracking. JACC Cardiovasc Interv 2010; 3: 155-64.

15. Yip HK, Chang HW, Wu CJ, et al. A safe and effective regimen without heparin therapy after successful primary coronary stenting in patients with acute myocardial infarction. Jpn Heart J 2000; 41: 697-711.

16. Fang HY, Chung SY, Sun CK, et al. Transradial and transbrachial arterial approach for simultaneous carotid angiographic examination and stenting using catheter looping and retrograde engagement technique. Ann Vasc Surg 2010; 24: 670-9.

17. Prasad A, Rihal CS, Lennon RJ, Wiste HJ, Singh M, Holmes DR Jr. Trends in outcomes after percutaneous coronary intervention for chronic total occlusions: a 25-year experience from the Mayo Clinic. J Am Coll Cardiol 2007; 49: 1611-8.

18. Saito S. Different strategies of retrograde approach in coronary angioplasty for chronic total occlusion. Catheter Cardiovasc Interv
2008; 71: 8-19.

19. Surmely JF, Tsuchikane E, Katoh O, et al. New concept for CTO recanalization using controlled antegrade and retrograde subintimal tracking: the CART technique. J Invasive Cardiol 2006; 18: 334-8.

20. Wu CJ, Lo PH, Chang KC, Fu M, Lau KW, Hung JS. Transradial coronary angiography and angioplasty in Chinese patients. Cathet Cardiovasc Diagn 1997; 40: 159-63.

21. Yip HK, Wu CJ, Chang HW, et al. Effect of the PercuSurge GuardWire device on the integrity of microvasculature and clinical outcomes during primary transradial coronary intervention in acute myocardial infarction. Am J Cardiol 2003; 92: 1331-5.

22. Cheng CI, Wu CJ, Fang CY, et al. Feasibility and safety of transradial stenting for unprotected left main coronary artery stenosis. Circ J 2007; 71: 855-61. 